\section{Mortalité des adultes infectés par le VIH comparée à la population générale}

\section{Données à long terme et selon les lymphocytes CD4}

Charlotte Lewden, Geneviève Chêne, Philippe Morlat, François Raffi, François Dabis, Catherine Leport et les groupes d'étude des cohortes ANRS C03 Aquitaine et ANRS CO8 Aproco-Copilote
C. Lewden : Inserm U897, Bordeaux, France ; ISPED, Université Victor Segalen Bordeaux 2, 146, rue Léo-Saignat, 33076 Bordeaux, France. G. Chêne, P. Morlat, F. Dabis : Inserm U897, Bordeaux, France; ISPED, Université Victor Segalen Bordeaux 2, 146, rue Léo-Saignat, 33076 Bordeaux, France. Centre Hospitalier Universitaire, Bordeaux, France. charlotte.lewden@isped.u-bordeaux2.fr F. Raffi : Université de Nantes, Nantes, France.

C. Leport: Université Paris 7 Denis Diderot, Paris, France.
$>$ Chez les personnes infectées par le VIH (virus immunodéficience humaine), la restauration immunitaire induite par les combinaisons thérapeutiques antirétrovirales disponibles à partir de 1996 a permis une forte diminution de l'incidence du Sida et de la mortalité [1]. Toutefois, la mortalité des adultes infectés par le VIH reste globalement supérieure à celle de la population générale de même âge et de même sexe [2]. Le marqueur le plus utilisé en pratique clinique pour mesurer l'immunité et son altération est le nombre de lymphocytes $\mathrm{T} \mathrm{CD4}^{+}$par $\mathrm{mm}^{3}$ (ou «CD4 »), qui baisse progressivement au cours de l'infection par le $\mathrm{VIH}$ en l'absence d'intervention et qui remonte sous traitement antirétroviral. Un nombre élevé de CD4 en début de traitement ou atteint après 6 mois de traitement est un facteur connu de meilleur pronostic [3]. Nous avons fait
I'hypothèse selon laquelle une certaine durée de traitement était nécessaire pour que la mortalité des personnes infectées par le VIH diminue jusqu'au niveau observé dans la population générale. Nous avons ainsi étudié la mortalité des personnes infectées par le VIH en fonction du nombre de lymphocytes CD4 atteint et de la durée du traitement antirétroviral [4].
Afin de disposer d'un recul suffisant, cette étude a été réalisée chez des adultes ayant débuté une combinaison de traitements antirétroviraux entre 1997 et 1999 et suivis dans deux cohortes françaises de l'Agence Nationale de Recherches sur le Sida et les hépatites virales (ANRS), la cohorte ANRS CO3 Aquitaine et la cohorte ANRS C08 Aproco-Copilote. La mortalité a

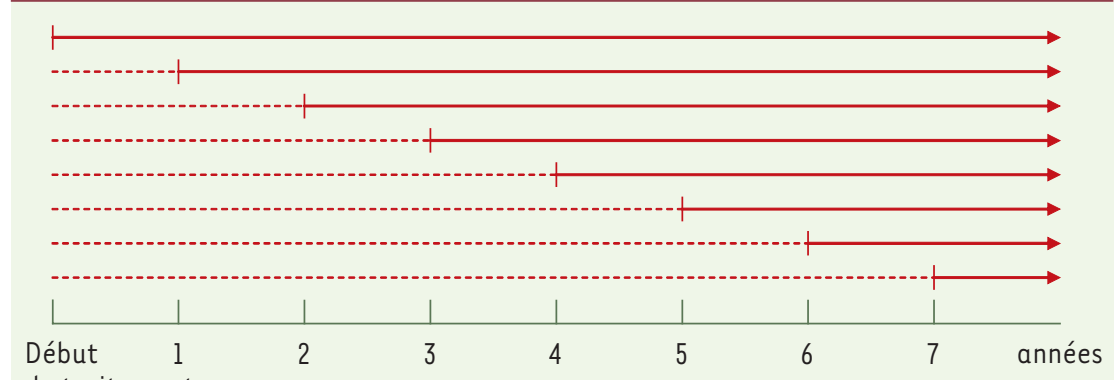

du traitement

Figure 1. Temps d'observation des analyses successives (trait plein) avec troncature à gauche à $1,2, \ldots 7$ ans de suivi. 
été comparée à celle de la population générale de même âge et de même sexe par l'estimation du rapport de mortalité standardisé SMR (standardized mortality ratio). Le SMR est le rapport entre le nombre de décès observé dans la population d'étude (ici l'ensemble des deux cohortes) et le nombre de décès qui serait attendu dans cette population si les taux de mortalité étaient ceux de la population générale. Si le SMR est égal à 1 (ou si son intervalle de confiance comprend la valeur 1), on conclut que la mortalité ne diffère pas entre la population d'où est issu l'échantillon étudié et la population générale. Pour étudier l'effet des CD4, des SMR ont été estimés séparément pour les périodes passées, et les nombres de CD4 classés dans chacune des catégories suivantes: moins de 200, 200 à 349, 350 à 499 et 500 cellules par $\mathrm{mm}^{3}$ ou plus. De plus, afin d'étudier si la mortalité pourrait atteindre celle de la population générale après une certaine durée de traitement, les SMR ont été estimés en tronquant les temps d'observation dans l'étude après $1,2, \ldots 7$ ans de traitement, comme indiqué dans la Figure 1. Par exemple, l'analyse avec troncature à 5 ans ne prend en compte que le temps passé après 5 ans de traitement (et sélectionne les person- nes avec au moins 5 ans de suivi après le début du traitement).

Un total de 2435 adultes infectés par le VIH traités par au moins 3 antirétroviraux ont été inclus dans l'étude, dont $77 \%$ d'hommes. Au début du traitement antirétroviral, la médiane de leur âge était de 36 ans et le nombre médian de CD4 était $270 / \mathrm{mm}^{3}$. La durée médiane de suivi pour cette analyse était de 7 ans. La mortalité était globalement 7 fois plus élevée que dans la population générale, 5 fois chez les hommes et 13 fois chez les femmes. Comme on pouvait s'y attendre, le SMR était d'autant plus bas que les nombres de CD4 étaient élevés. Chez les personnes ayant des CD4 supérieurs à $500 / \mathrm{mm}^{3}$, la mortalité était plus élevée que dans la population générale si l'on considérait l'ensemble du suivi, mais elle devenait comparable à celle de la population générale après 6 ans de suivi sous traitement (Figure 2).

Dans cette étude, les personnes dont la mortalité a baissé jusqu'à atteindre les taux observés dans la population générale sont celles qui ont atteint une réponse immunologique avec des CD4 supérieurs à $500 / \mathrm{mm}^{3}$, et qui ont été suivies et traitées de façon prolongée. Il s'agit bien d'une sélection particulière d'individus parmi les personnes infectées par le VIH qui ne représente pas les patients en échec de traitement ou ceux dont le suivi est interrompu. Mais ces résultats montrent qu'il existe des conditions dans lesquelles la mortalité a diminué au niveau de celle de la population générale, et cela permet de définir pour l'ensemble des patients des objectifs de restauration de l'immunité, et d'inciter les équipes médicales et les patients à évaluer et surmonter les obstacles à une réponse optimale au traitement. Les personnes qui ont participé à cette étude ont débuté un traitement à la fin des années 1990 , et de nouvelles molécules plus efficaces et mieux tolérées ont été progressivement disponibles depuis. Il est donc possible que la durée des traitements antirétroviraux nécessaire pour obtenir une telle diminution de la mortalité soit actuellement inférieure à 6 ans. Enfin, bien que les taux de mortalité des personnes infectées par le VIH ne diffèrent pas entre les femmes et les hommes, le SMR est plus élevé chez les femmes que chez les hommes. Cela s'explique par le fait que, dans la population générale utilisée comme référence, la mortalité dans les classes d'âges étudiées est plus faible chez les femmes que chez les hommes.

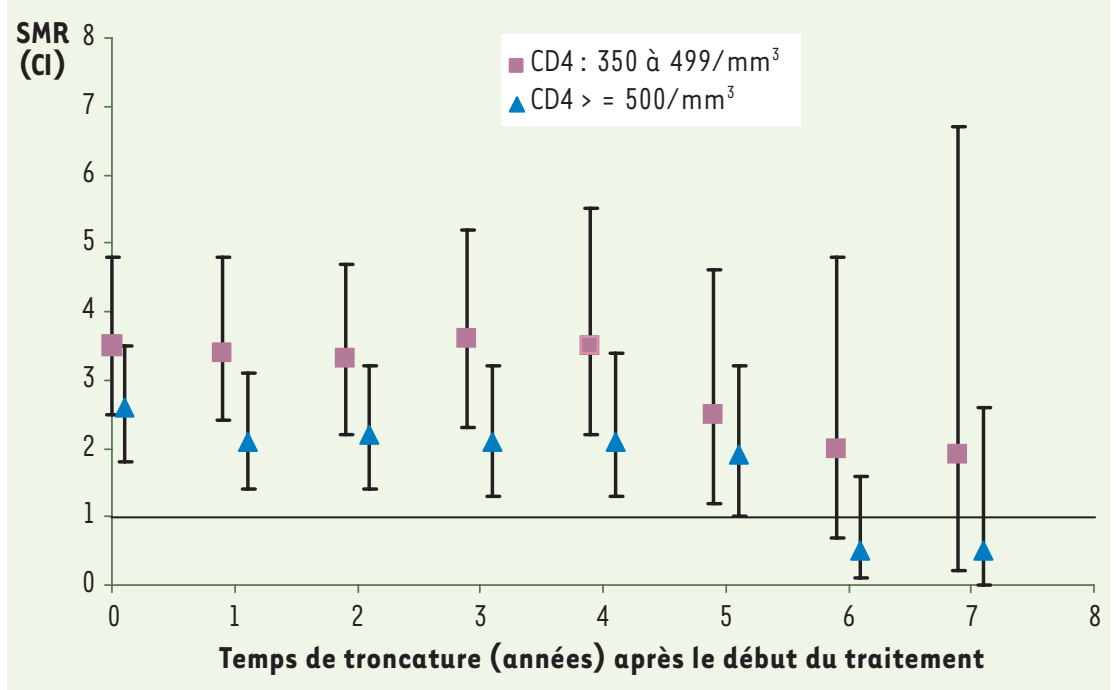

Figure 2. Standardized Mortality Ratios (SMR) selon le temps passé avec des lymphocytes $T$ CD4 entre 350 et $499 / \mathrm{mm}^{3}$ et au-dessus de $500 / \mathrm{mm}^{3}$, et selon le temps de troncature. Cette figure représente les SMR pour les personnes infectées par le VIH avec plus de 500 $\mathrm{CD} 4 / \mathrm{mm}^{3}$ et avec 350 à $499 \mathrm{CD} 4 / \mathrm{mm}^{3}$, selon le moment où on a débuté l'observation (troncature à $1,2, \ldots 7$ ans après le début du traitement antirétroviral). Seuls les SMR estimés lorsque les CD4 sont supérieurs à $500 / \mathrm{mm}^{3}$ et après 6 ans de suivi se rapprochent de la valeur 1 , qui correspond à une mortalité similaire à la population générale (ANRS $\mathrm{CO} 3$ Aquitaine et ANRS C08 Aproco-Copilote). 
Ces résultats sont concordants avec d'autres études, comme l'étude internationale DAD qui a rapporté une diminution des taux de mortalité avec l'augmentation des CD4, y compris au-dessus de 350 et 500 $\mathrm{CD} 4 / \mathrm{mm}^{3}$ [5]. Ils plaident pour que les personnes infectées par le VIH soient traitées avec des objectifs de restauration immunitaire exigeants avec un nombre de CD4 supérieur à $500 \mathrm{CD} 4 / \mathrm{mm}^{3}$. Indirectement, ils incitent à une initiation précoce du traitement antirétroviral afin de faciliter l'atteinte de cet objectif [6]. Les recommandations actuelles de début de traitement antirétroviral retiennent le seuil de $350 / \mathrm{mm}^{3}$ [7], mais une mise en route plus précoce est discutée et est mise en balance avec les inconvénients d'un traitement très précoce, dont les effets secondaires $[10,11]$ et le risque d'épuisement des ressources thérapeutiques $[8,9]$. En conclusion, cette étude souligne le chemin parcouru depuis le début de l'épidémie d'infection par le VIH et elle est source d'espoir et d'encouragements pour les personnes qui suivent actuellement un traitement antirétroviral. $\diamond$

Mortality rate of HIV-infected adults on long term combination antiretroviral therapy

\section{RÉFÉRENCES}

1. CASCADE Collaboration. Determinants of survival following HIV-1 seroconversion after the introduction of HAART. Lancet $2003 ; 362$ : 1267-74.

2. Van Sighem A, Danner $S$, Ghani AC, et al. Mortality in patients with successful initial response to highly active antiretroviral therapy is still higher than in non-HIV-Infected individuals. J Acquir Immune Defic Syndr 2005; $40: 212-8$.

3. Chêne G, Sterne JA, May M, et al. Prognostic importance of initial response in HIV-l infected patients starting potent antiretroviral therapy: analysis of prospective studies. Lancet 2003 ; 362: 679-86.

4. Lewden C, Chêne G, Morlat P, et al. HIV-infected adults with a CD4 cell count greater than 500 cells/ $\mathrm{mm}^{3}$ on long term combination antiretroviral therapy reach same mortality rates as the general population. J Acquir Immune Defic Syndr 2007 ; 46 : $72-7$.

5. The data collection on adverse events of anti-HIV drugs study group. Liver-related deaths in persons infected with the human immunodeficiency virus : the
DAD study. Arch Intern Med 2006; 166 : 1632-41.

6. Gras L, Kesselring AM, Griffin JT, et al. CD4 Cell counts of 800 cells $/ \mathrm{mm} 3$ or greater after 7 years of highly active antiretroviral therapy are feasible in most patients starting with 350 cells $/ \mathrm{mm} 3$ or greater. J Acquir Immune Defic Syndr 2007 ; 45 : 183-92.

7. Yéni. Prise en charge médicale des personnes infectées par le VIH. Rapport 2006. Recommandations du groupe d'experts. 2006 [cited 2007 march 2007]. Available from : http://www.sante.gouv.fr/htm/actu/ yeni_sida/rapport_experts_2006.pdf

8. Phillips AN, Gazzard BG, Clumeck N, Losso MH, Lundgren JD. When should antiretroviral therapy for HIV be started? Br Med / 2007 ; 334 : 76-8.
9. The DAD Study Group. Class of antiretroviral drugs and the risk of myocardial infarction. $N$ Engl J Med 2007; 356: 1723-35.

10. Capeau J, Caron M, Vigouroux C, et al. Les lipodystrophies secondaires aux traitements antirétroviraux de l'infection par le VIH. Med Sci (Paris) 2006; $22: 531-6$.

11. Moatti JP, Spire B. Les enjeux économiques et sociaux de l'infection par le VIH à l'ère des multithérapies antirétrovirales : apports des sciences humaines et sociales. Med Sci (Paris) : 2003 ; 19 : 878-84.
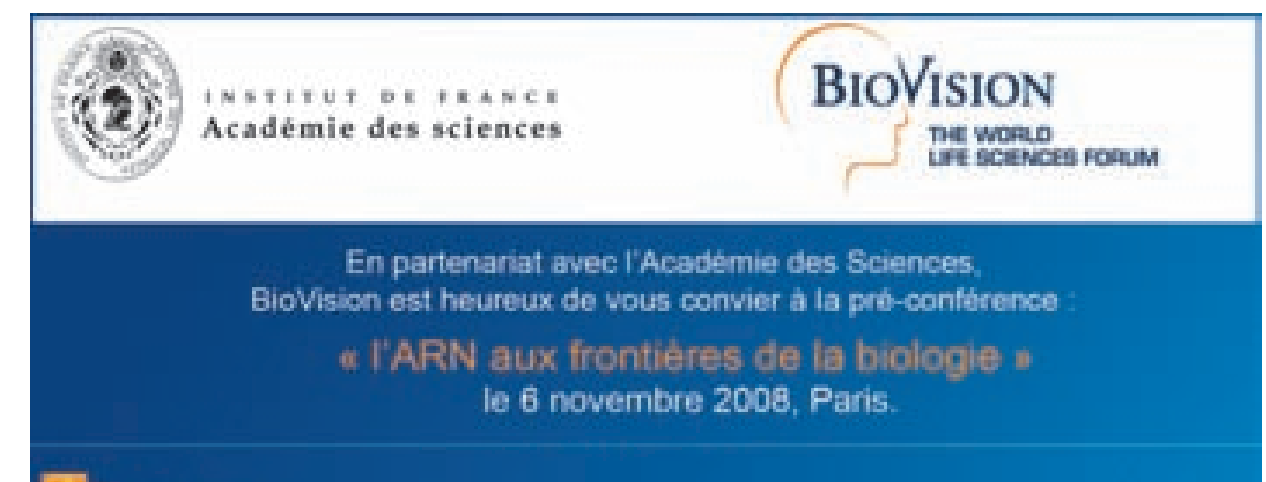

Programme

10:30 - 10:45 - Introduction

- Dr Françis Gros, Academie des Sciences

10:45 - 13:00 - Interventions

- Pror Wirold Filpowicz, Friedrich Miescher Institute for Biomedical Research

- Dr Oviver Voinnet, Insasat de Biologie Mosiculaire des Plantes du CNRS

- Dr Edith Heard Institut Curie

13:00 - Dejeuner
$14: 15-16: 30-$ interventiant

- Prof Jeachim Lingner, Swiss Instatute for Experimental Cancer Research

- Dr Pascale Romby. IBMC-CNRs

- Tharmo Fisher scientific

16:30 - 17:00-Conctusions

- Prof Enc Whstrof, Acadtmie des Sciences
7. Leu :

Fondation Cino del Luca, Institut de France to rue Altred de Viany 75006 PARIS Tol : $+33(0) 147660121$

\section{Organisation :}

Prof Eric Westhof, Insthut de biologie moléculaire et cellulaire du CNRS
Inscription obligatoire : E-mail ; d werlinggibme.u-strasbg.ft Tel : +33 (0) 388417056 Fax: $+33(0) 388602218$ (aupres du secreftariat de lunite)

\section{BIOVISION, LE 6 F FORUM MONDIAL DES SCIENCES DE LAVIE Lyon, 8 - -11 mars 2009}

BioVision - Le Forum Mondal des Scienses de in Vie est une plateloime inteinaticnale de dialogue, de debats et de propositions dactions concretes reunisaant des iepresentants be la science, de la societe clyle et de rndustre, pour travaller ensemble suif les prands enjeur de santh, dagiculture et de nutition, demironnement et dienespe. 